\title{
O papel da Universidade Aberta à Terceira Idade na educação ambiental
}

The role of the University of the Third Age at protecting the natural environment El papel de la Universidad de la Tercera Edad en la protección ambiental

\author{
Ronise Straiotto PIATO ${ }^{1}$ \\ Letícia Cabrera CAPALBO ${ }^{1}$ \\ Maria Isabel Rosifini ALVES REZENDE ${ }^{2}$ \\ Lucas de Souza LEHFELD ${ }^{3}$ \\ Maria Cristina Rosifini Alves ALVES REZENDE ${ }^{4}$
}

${ }^{1}$ Graduação em Odontologia, Faculdade de Odontologia de Araçatuba, Universidade Estadual Paulista “Júlio de Mesquita Filho”, UNESP, Brasil
'Graduação em Direito, Faculdade Laudo de Camargo, Universidade de Ribeirão Preto, Brasil
${ }^{3}$ Pós-Doutor em Direito pela Universidade de Coimbra (UC). Doutor em Direito pela PUC/SP.. Avaliador de Cursos de Direito pelo INEP/MEC,
Docente do Curso de Mestrado da Universidade de Ribeirão Preto, Brasil
${ }^{4}$ Professora Adjunto, Faculdade de Odontologia de Araçatuba, Universidade Estadual Paulista “Júlio de Mesquita Filho”, UNESP, Brasil

\section{Resumo}

Envelhecimento populacional e proteção ambiental vêm ganhando especial relevância, tendo em vista as recentes transformações econômicas, demográficas e sociais no conjunto da sociedade. Objetivou-se promover revisão teórica e reflexiva com ênfase na convergência entre desenvolvimento sustentável e ações educacionais promovidas pela Universidade Aberta à Terceira Idade. Realizou-se revisão da literatura obtendo-se artigos na íntegra a partir dos descritores idoso, indicadores de desenvolvimento sustentável e proteção ambiental, publicados em inglês, espanhol e português, entre janeiro de 1994 e junho de 2014, em periódicos nacionais e internacionais nas bases Lillacs, Bireme e Medline. Utilizou-se roteiro sistematizado de coleta de dados para análise da amostra. Os resultados indicam que a Universidade Aberta à Terceira Idade desenvolve atividades de ensino, pesquisa e extensão, através de ações baseadas em pressupostos gerontológicos sócioeducativos. Neste contexto, a educação ambiental se apresenta como processo pedagógico capaz de despertar a capacidade crítica do idoso, tornando-o agente social ativo e conduzindo-o a uma concepção cultural que assegure estabelecimento de relações harmônicas sustentáveis com a natureza. Concluiu-se que a Universidade Aberta à Terceira Idade contribui para iniciativas que privilegiam as relações entre sistemas e processos econômicos, sociais e naturais, gerando a construção de valores sociais, conhecimentos, habilidades, atitudes e competências voltadas para a conservação do meio ambiente.

Descritores: Idoso; Universidades; Indicadores de Desenvolvimento Sustentável; Educação Ambiental.

\section{Abstract}

Population aging and environmental protection have gained special importance after the economic, demographic and social recent changes. The objective of this paper was to promote theoretical and reflective review with emphasis on convergence between sustainable development and educational activities promoted by the University of the Third Age. We conducted a literature review to give full articles from the descriptors: aged, sustainable development indicators and environmental protection, published in English, Spanish and Portuguese, between January 1994 and June 2014, in national and international journals on the basis Lillacs, Bireme and Medline. We used systematic data collection for sample analysis. The results indicate that the University of the Third Age develops teaching, research and extension, through actions based on aging and educational assumptions. In this context, environmental education is presented as a pedagogical process capable of awakening the critical capacity of the elderly, making it active social worker and leading him to a cultural design which ensures establishment of sustainable harmonious relationship with nature. It was concluded that the University of the Third Age contributes to initiatives that emphasize the relationships between systems and economic processes or social and natural processes, generating the construction of social values, knowledge, skills, attitudes and skills aimed at the conservation of the environment.

Descriptors: Aged; Universities; Sustainable Development Indicators; Environmental Education.

\section{Resumen}

Envejecimiento de la población y la protección del medio ambiente han adquirido especial importancia después de los cambios económicos, demográficos y sociales recientes. El objetivo de este trabajo fue promover revisión teórica y reflexiva, con énfasis en la convergencia entre el desarrollo sostenible y las actividades educativas promovidas por la Universidad de la Tercera Edad. Se realizó una revisión de la literatura de artículos completos. Los descriptores utilizados fueran: anciano, indicadores de desarrollo sostenible de edad y protección ambiental. El artículos fueran publicados en revistas nacionales e internacionales en los idiomas inglés, español y portugués, entre enero de 1994 y junio de 2014 y indexados en las bases: LiIlacs, Bireme y Medline. Utilizamos recopilación sistemática de datos para el análisis de la muestra. Los resultados indican que la Universidad de la Tercera Edad desarrolla la docencia, investigación y extensión, a través de acciones basadas en el envejecimiento y supuestos educativos. En este contexto, la educación ambiental se presenta como un proceso pedagógico capaz de despertar la capacidad crítica de los ancianos, con el establecimiento de una relación armoniosa con la naturaleza sostenible. Se concluyó que la Universidad de la Tercera Edad contribuye para las iniciativas que hacen hincapié en las relaciones entre los sistemas, los procesos económicos, los procesos sociales y los procesos naturales, generando la construcción de valores sociales, conocimientos, habilidades, actitudes y habilidades marcadas en la conservación del medio ambiente.

Descriptores: Ancianos; Universidades; Indicadores de Desarrollo Sostenible; Educación Ambiental.

\section{INTRODUÇÃO}

Envelhecimento populacional e proteção ambiental vêm ganhando especial relevância, tendo em vista as recentes transformações econômicas, demográficas e sociais no conjunto da sociedade ${ }^{1,2}$. $\mathrm{Na}$ atualidade, é aceito que o potencial evolutivo da humanidade está assegurado na medida em que os recursos naturais são preservados. A própria Constituição Federal de 1988, ao elevar o meio ambiente à categoria dos bens tutelados pelo ordenamento jurídico, sistematizou a matéria ambiental e estabeleceu o direito ao meio ambiente sadio como um direito fundamental do indivíduo ${ }^{2}$.

Neste contexto, o direito ambiental traz em seu bojo uma postura ética do homem frente à preservação da qualidade dos ecossistemas e à valorização da biodiversidade ${ }^{3}$, alicerçada na equidade intergeracional. Entende-se assim o meio ambiente como consequência do envolvimento de todos os seres vivos com o planeta e a defesa do ambiente saudável como dever e direito inalienável 
das gerações atual e futura ${ }^{3,4}$. Significa dizer que a presente geração não pode, em absoluto, usufruir de todo o recurso fornecido pelo meio ambiente de modo a deixar para as próximas gerações um saldo mínimo ou insuficiente ${ }^{2}$.

Um ambiente sadio é, portanto, um direito de todo ser vivo. E o direito ao meio ambiente decorre do mais fundamental de todos os direitos de um Estado democrático - o direito à vida e à existência, garantido constitucionalmente no artigo $5^{\circ}$ que estabelece os direitos e deveres fundamentais do homem, definindo as regras de organização da sociedade e impondo seus limites 5 .

A origem do homem se confunde com o próprio processo de degradação do planeta. A proporção do dano causado ao meio ambiente é o que diferencia a utilização dos recursos naturais pelo homem primitivo ${ }^{3}$.

A Universidade consagra-se, desde a sua criação na idade média, por papéis e funções diferenciados, em decorrência das ligações e subordinações pelas quais passou em cada momento histórico ${ }^{6}$.

A universidade tem um forte compromisso com a transformação da sociedade, com o exercício da crítica livre, com a preservação do conhecimento, com a construção de um novo saber, com a beleza, com as artes e com a cultura, alicerçada nos valores da ética da democracia, da justiça e da igualdade que norteiam a sociedade ${ }^{6}$.

Por ser instrumento de formação e desenvolvimento de indivíduos, também cabe à Universidade a criação das bases para o desenvolvimento humano e ambiental sustentáveis. O crescimento de programas universitários conhecidos como Universidades Abertas à Terceira Idade (UATIS ou UnATIS), traduzem o interesse $\mathrm{e}$ motivação da população idosa por conhecimento, interação social, atividades recreativas e de lazer?

O propósito deste trabalho é promover revisão teórica e reflexiva com ênfase na convergência entre desenvolvimento sustentável e ações educacionais promovidas pelas Universidades Abertas à Terceira Idade.

\section{MATERIAL E MÉTODO}

Realizou-se revisão da literatura obtendo-se artigos a partir dos descritores idoso, universidades, indicadores de desenvolvimento sustentável e educação ambiental, publicados em inglês, espanhol e português, entre janeiro de 1994 e junho de 2014, em periódicos nacionais e internacionais nas bases Lillacs, Bireme e Medline. Utilizou-se roteiro sistematizado de coleta de dados para análise da amostra: pesquisa do material, leitura dos artigos e seleção dos textos, totalizando 37 trabalhos.

As seguintes etapas foram percorridas: estabelecimento da hipótese e objetivos do estudo; estabelecimento de critérios de inclusão e exclusão de artigos (seleção da amostra), tomando-se como guia da revisão a seguinte questão: a Universidade Aberta à Terceira Idade tem papel sobre a educação ambiental?

Como critérios de elegibilidade e inclusão dos artigos, analisaram-se a procedência da revista e indexação, estudos que apresentassem dados referentes ao idoso, universidades, indicadores de desenvolvimento sustentável e educação ambiental, publicados entre janeiro de 1994 e junho de 2014. Na leitura e avaliação, os artigos que apresentaram os critérios de elegibilidade foram selecionados e incluídos na pesquisa por consenso.

\section{RESULTADOS E DISCUSSÃO}

\section{- O surgimento da Universidade Aberta à Terceira Idade}

A primeira Universidade da Terceira Idade (U3As), l'Université du troisième âge, foi criada em Toulouse, França, em 1972. Afiliada ao campus universitário oferecia aos idosos programas intelectuais e artísticos, de lazer e atividades físicas ${ }^{8}$. De acordo com o modelo francês, as oportunidades oferecidas pela sociedade aos idosos eram quase inexistentes e a partir disso, a universidade deveria se abrir a esta faixa etária ${ }^{9,10}$. Muitos U3As na Europa seguiram o modelo francês, oferecendo cursos, seminários, sessões e discussões abertas com base nas necessidades e interesses dos idosos da comunidade $^{11,12}$.

Em 1981, a versão britânica da U3A foi criada em Cambridge com perfil baseado na autoajuda e autossuficiência ${ }^{13}$, não afiliada à universidade tradicional, formada por um corpo de indivíduos que se comprometiam a aprender e ajudar os outros a aprenderem ${ }^{14-16}$. O modelo U3A Britânica foi adotado na Austrália, Nova Zelândia e África do Sul.

No Brasil, em 1992 foi criada na Universidade Estadual do Rio de Janeiro (UERJ) a Universidade Aberta à Terceira Idade (UnATIUERJ), nos moldes do modelo francês e com o propósito de: 1) trazer à população idosa uma alternativa para utilização de seu tempo livre de maneira cultural, social e esportiva; 2) permitir a integração intergeracional; 3) permitir a atualização e aquisição de novos conhecimentos, possibilitando a participação integral, a elevação da autoestima e, como consequência, contribuir para uma melhora significativa da qualidade de vida dos idosos ${ }^{7}$.

Para Neto $^{17}$ a criação no Brasil da Universidade da Terceira Idade, Universidade Aberta à Terceira Idade ou ainda, Universidade para a Terceira Idade trouxe aos idosos espaço social legítimo, um locus onde estar bem com a vida e com a idade passou a ser vivido coletivamente, favorecendo $\mathrm{o}$ fortalecimento de vínculos, o 
aprendizado mútuo, a conquista de novas amizades e a formação de redes de apoio.

$\mathrm{O}$ êxito alcançado no contexto brasileiro propiciou uma maior visibilidade das questões referentes ao idoso e ao envelhecimento populacional demográfico, confirmando a função social da Universidade e seu comprometimento com as demandas da comunidade ${ }^{18}$.

\section{- Universidade X educação ambiental X idoso}

As experiências no passado, dos indivíduos que na atualidade formam o grupo com idade acima de 60 anos ou mais, também contribuíram para a preservação ou a deterioração do meio ambiente ${ }^{19}$.

A Educação Sanitária Ambiental na visão de Pinheiro et al. $^{20}$ adquire caráter estratégico na proteção ambiental ao privilegiar a participação da população na busca de soluções viáveis, integrando o conhecimento sistematizado e a realidade dos partícipes sociais envolvidos, levando à sensibilização, comprometimento e consciência da proteção ambiental.

Tristão $^{22}$ lembra que a efetivação das políticas para ações sustentáveis está diretamente relacionada a uma nova racionalidade, alicerçada na postura responsável das gerações atuais e futuras e nas atitudes dos atores sociais.

Luzzi $^{23}$ ressalta que a educação se modifica ao longo do processo histórico em resposta ao diálogo de um conjunto de forças sociais em conflito, as quais expressam concepções sobre 0 conhecimento, a aprendizagem, a sociedade e o ambiente natural.

Miranda et al. ${ }^{24}$ destacam que a educação ambiental busca a construção de valores, conceitos, habilidades e atitudes que permitam o entendimento da realidade da vida e a atuação lúcida e responsável de atores sociais, individuais e coletivos, no ambiente.

Para Guimarães ${ }^{25}$ a educação ambiental mostra-se potencialmente capaz de estimular a solidariedade, a igualdade e o respeito aos direitos humanos, valendo-se de estratégias democráticas e interação entre as culturas.

Segundo Mayor $^{26}$ a educação se apresenta como a chave do desenvolvimento sustentável e autossuficiente. Dessa forma, deve ser fornecida igualitariamente a todos os membros da sociedade, de tal maneira que cada um se beneficie de chances reais de se instruir ao longo da vida.

A cultura ambiental fomentada na universidade é potencialmente capaz de se refletir positiva e rapidamente na sociedade, por meio do desenvolvimento da cidadania ambiental e da ética ecológica $^{27-29}$. Espera-se comportamento social e ambiental responsáveis da comunidade acadêmica, com melhoria das habilidades, valores e competências para si e para os grupos sobre os quais exerce influência.
O ambiente na Universidade Aberta á Terceira Idade é fértil ao alimentar debates transformadores, capazes de levar conhecimentos, habilidades e novos valores para o cotidiano do idoso, contribuindo para a melhoria das comunidades onde estão inseridos ${ }^{30}$.

A sociedade moderna está focada no modelo capitalista, na valorização da competitividade, na capacidade para o trabalho, na independência e na autonomia funciona ${ }^{31,32}$. Nesse contexto, o indivíduo idoso com suas perdas físicas, emocionais e sociais sofre cruel marginalização. A representação social da terceira idade após a Revolução Industrial tornou-se fortemente atrelada ao conceito de declínio ${ }^{33}$, apartação social e exclusão ${ }^{34-37}$.

$\mathrm{O}$ entendimento de que o comprometimento da população idosa passa por mecanismos ativos de educação continuada com expressiva melhoria do desempenho ambiental reforça o papel da Universidade Aberta à Terceira Idade na conscientização do indivíduo idoso de que além de destinatário de direitos também é titular de deveres diante do meio ambiente.

\section{CONCLUSÃO}

As relações entre a terceira idade e o meio ambiente se reproduzem de maneira complexa e problemática nos contextos sociais vulneráveis, especialmente próprios da realidade do idoso brasileiro.

O sucesso do desenvolvimento sustentável está estreitamente relacionado com a participação de todos os indivíduos e seu empenho como sujeitos ativos na proteção do ambiente.

A Universidade Aberta à Terceira Idade, por sua posição pautada, sobretudo no processo de educação continuada, contribui para exteriorização da consciência ambientalista junto à população idosa, adquirida pelo reconhecimento do direito fundamental, aos quais estão coobrigados, isto é, reconhecimento de que são sujeitos ativos do dever na manutenção de um ambiente saudável.

\section{REFERÊNCIAS}

1. Gico VV, Carvalho MOF. A participação do idoso na educação ambiental como exercício da sua cidadania. InterScientia. 2014;2(2):56-76.

2. Silva RMP. O meio ambiente na Constituição Federal de $1988 . \quad$ Disponível em http://jus.com.br/artigos/25529. Acesso em 14 de junho de 2014.

3. Sparemberguer RFL, Silva DA. A relação homem, meio ambiente, desenvolvimento e o papel do direito ambiental. Disponível em http:// www.domtotal.com/ direito/ pagina/detalhe/ 23711. Acesso em 10 de junho de 2014.

4. Rocha TA, Queiroz MOB. O meio ambiente como um direito fundamental da pessoa humana. 
Disponível em http://ambitojuridico.com. br/ site/? artigo_id $=10795 \& \mathrm{n}_{-} \quad$ link= revista artigos_leitura

5. Silva TC. O meio ambiente na Constituição Federal de 1988. 2008. Disponível em http://www.jurisway.org.br/v2/ dhall.asp? id_dh=940 >. Acesso em 18 de fevereiro de 2014.

6. Siqueira AC. As novas relações entre a universidade e a sociedade brasileira na era da revolução científico-tecnológica: o saber (poder) em disputa. Disponível em http://www.anped11 uerj.br/ 18/SIQUEIRA.htm. Acesso em 05 de abril de 2014.

7. Veras R, Caldas C. UnATI-UERJ-10 anos. Um modelo de cuidado integral para a população que envelhece. Rio de Janeiro: RelumeDumará/UnATI/UERJ; 2004.

8. Glendenning F. Education for older adults. Int $J$ Lifelong Educ. 2001;20(1/2): 63-70.

9. Vellas P. University of the Third Age. AIUTA Association Internationale des Universités du Troisième Age. Disponível em: www.worldu3a.org/ worldpapers /vellas-uk.htm. Acesso em 01 de maio de 2014.

10. Hebestreit L. The role of the University of the Third Age. Aust J Adult Learn. 2008;48(3):54765.

11.Formosa M. Older adult education in a Maltese University of the Third Age: a critical perspective. Education and Ageing. 2000;15(3): 315-39.

12.Formosa M. Critical gerogogy: developing practical possibilities for critical educational gerontology. Int J Educ Ageing. 2002; 17(3):7386

13.Laslett P. A fresh map of life: the emergence of the Third Age, 2 ed. London: Macmillan Press; 1996.

14.Brady EM, Holt SR, Welt B. Peer teaching in lifelong learning institutes, J Educ Gerontol. 2003;29(10): 851-68.

15. Manheimer RJ. Older adult education in the United States: trends and predictions, North Carolina Center for Creative Retirement, University of North Carolina, Ashville. Disponível em http://www.unca.edu. Acesso em 01 de abril de 2014.

16. Midwinter E. Happy anniversary! U3A Sources. An Educational Bulletin. 2003; 19(6): 1-2.

17. Neto AJ. A Universidade Aberta para terceira Idade da PUC -SP. In: A terceira Idade. São Paulo: SESC, 14ed.1998.

18. Silva FL. Reflexões sobre o conceito e a função da universidade pública. Estudos Avançados. 2001; 15 (42):295-304.

19. Machado PAL. Direito Ambiental Brasileiro. 10. ed. São Paulo: Malheiros, 2002.

20.Pinheiro JI, Lima BUM, Dantas Jr. PC. A educação sanitária e ambiental como instrumento de participação popular, conscientização e controle social na regulação dos serviços de saneamento ambiental. Disponível em https://www.natal.rn.gov.br/

arsban/paginas/File/A_EDUCACAO_SANITARI A_AMBIENTAL_COMO_INSTRUMENTO.pdf. Acesso em 01 de março de 2014.

21.Bittar M, Pereira KAB, Grigoli JAG. Educação ambiental e universidade: algumas considerações sobre a formação de professores. Disponível em: http://www.histedbr.fe. acer_histedbr/jornada/ unicamp.br/ jornada7/ _GT4\%20PDF/EDUCA\%C7\%C3O\%20AMBIEN TAL\%20E\%20UNIVERSIDADE\%20GT4.pdfAc esso em 05 de maio de 2014.

22.Tristão M. A educação ambiental na formação de professores: redes de saberes. São Paulo: Annablume; 2004.

23.Luzzi D. A "ambientalização" da educação formal. Um diálogo aberto na complexidade do campo educativo. In Leff H.(org.). A complexidade ambiental. Trad. Eliete Wolff. São Paulo: Cortez; 2003. p. 178-216.

24.Miranda ES, Schall VT, Modena CM. Representações sociais sobre educação ambiental em grupos da terceira idade. Ciênc Educ. 2007; 13(1):15-28.

25.Guimarães M. Educação Ambiental Crítica. In: Layrargues PP (Coord.). Identidades da Educação Brasileira. Brasília: Ministério do Meio Ambiente, 2004.

26. Mayor F. Preparar um futuro viável: ensino superior e desenvolvimento sustentável. In: Conferência mundial sobre o ensino superior. Tendências de educação superior para o século XXI. Anais. Paris: 1998.

27.Nunes EM. A educação ambiental na universidade: caminhos e possibilidades para a sustentabilidade ecológica. Disponível em http://www.ecossistemica.com.br/reflexoes/Educa caoambientaleuniversidade.pdf. Acesso em 15 de maio de 2014.

28. Salgado MFMA, Cantarino AAA. O papel das instituições de ensino superior na formação socioambiental dos futuros profissionais. ENEGEP. 2006;26:1-8.

29. Fouto ARF. O papel das universidades rumo ao desenvolvimento sustentável: das relações internacionais às práticas locais. Disponível em http://campus.fct.unl.pt/ campus verde/ W_RIA_ARFF.doc. Acesso em 08 março 2014.

30. Toni IAM. As instituições de endino superior e as UNATIS Brasileiras. In: Rita de Cássia da Silva de Oliveira; Raimunda Silva D' Alencar. (Org.). As esperiências de universidades abertas em um Brasil que envelhece. CRV.2011;1:161-74.

31. Bueno GDR, Roseli VR. A Importância da Universidade Aberta da Terceira Idade: 
Unati/Unicentro. Disponível em http://www.isapg.com.br/2011/ciepg/download.ph p?id=37. Acesso em 20 de março de 2014.

32. Veloz MCT, Nascimento-Schulze CM, Camargo BV. Representações sociais do envelhecimento. Psicol Reflex Crit. 1999; 12(2):479-501.

33. Goldfarb DC. Corpo, tempo e Envelhecimento. São Paulo: Casa do Psicólogo; 1998.

34.Alves-Rezende MCR, Capalbo BC, Soubhia AMP, Poi WR, Fajardo RS, Bresciani KDS, Fonseca LEC. UNATI - promoção de qualidade de vida na terceira idade. Arch Health Invest. 2012; 1(Supl 1):64.

35. Capalbo LC, Piato RS, Fajardo RS, Rezende MIRA, Lehfeld LS, Rezende MCRA. Representação social da velhice e ações promovidas pela Universidade: responsabilidade social da Unati. Rev Odontol UNESP. 2014; 43(N Especial):112.

36. Alves-Rezende MCR, Momesso GAC, Pupim LF, Brasilino MS, Pires-Soubhia AM, Poi WR, Bresciani KD, Fonseca LEC, Fajardo RS. Arteterapia: motivação pessoal na terceira idade. Arch Health Invest. 2012; 1(Supl 1):16.

37. Alves Rezende MCR, Bispo ACO. Saúde bucal na terceira idade: egressos do curso de odontologia frente às mudanças nos modelos de atuação pública e privada. Rev Reg Araçatuba Assoc Paul Cir Dent. 2001; 22(2):1-6. 


\section{CONFLITO DE INTERESSES}

Os autores declaram não haver conflitos de interesse.

AUTOR PARA CORRESPONDENCIA

\section{Maria Cristina Rosifini Alves Rezende}

rezende@foa.unesp.br

Submetido em 01/07/2014

Aceito em 21/07/2014 